\title{
We need to predict extubation failure
}

\author{
É preciso prever a falha de extubação
}

\author{
Julio A. Farias, ${ }^{1}$ Ezequiel Monteverde ${ }^{2}$
}

$\bigcup_{m}$ de cada três pacientes pediátricos admitidos à unidade de terapia intensiva (UTI) pediátrica irá exigir suporte respiratório por uma média de 5 dias $^{1}$. Mesmo diante dos benefícios universalmente aceitos da ventilação mecânica (VM) em crianças com insuficiência respiratória, os riscos associados a essa prática exigem que a equipe de cuidado intensivo busque constantemente compreender melhor a fisiopatologia dessa situação especial e desenvolver índices ou parâmetros que possam embasar a decisão de interromper a VM o mais cedo possível. O espectro da falha do desmame ronda a cena, enquanto, ao mesmo tempo, os médicos se deparam com a ameaça de prolongar desnecessariamente a VM.

$A$ retirada da $V M$ se refere à transferência, rápida ou gradativa, do esforço respiratório do ventilador para o paciente (desmame), enquanto que a extubação se refere à remoção da cânula endotraqueal. Freqüentemente encarada como uma continuação natural do desmame, a extubação tem suas próprias características e fatores preditivos de desfecho, que levam em consideração, principalmente, a capacidade de oferecer proteção à via aérea, o manejo de secreções e a desobstrução das vias aéreas superiores ${ }^{2}$.

Cinqüenta e cinco estudos, totalizando aproximadamente 33.000 pacientes, demonstraram que $12.5 \%$ (intervalo: $2-25 \%$ ) dos pacientes adultos extubados exigem reintubação 24-72 h depois da remoção da cânula endo-

1. MD. Jefe, Unidad de Cuidados Intensivos Pediátricos, Departamento de Urgencia, Hospital de Niños R Gutiérrez, Universidad de Buenos Aires, Buenos Aires, Argentina.

2. MD. Médico residente, Unidad de Cuidados Intensivos Pediátricos, Departamento de Urgencia, Hospital de Niños R Gutiérrez, Universidad de Buenos Aires, Buenos Aires, Argentina.

Como citar este artigo: Farias JA, Monteverde E. We need to predict extubation failure. J Pediatr (Rio J). 2006;82:322-4.

doi:10.2223/JPED.1539 traqueal $^{2}$. Da mesma forma, o intervalo de falha de extubação em crianças é bastante heterogêneo, variando de 4,9 a 29\%1,3-11. A taxa ótima de falha de extubação na UTI pediátrica permanece controversa. De um lado, a extubação injustificadamente tardia está associada a um tempo maior de permanência na UTI, maior risco de pneumonia associada à ventilação e maior mortalidade. De outro lado, a interrupção prematura da ventilação está intimamente relacionada à falha de extubação e à necessidade de reintubação, com desfechos adversos que incluem hospitalização prolongada, custos mais elevados, maior necessidade de traqueotomia e, em alguns estudos, maior mortalida$\mathrm{de}^{1,8,10-12}$. Na ausência de um consenso claro do que seja uma taxa ideal de falha de extubação, os médicos precisam refletir sobre a melhor escolha entre limitar o período de VM ou minimizar a falha de extubação.

Os resultados negativos da extubação tanto tardia quanto prematura, assim como a recente informação de que a ventilação não-invasiva pode ser ineficaz nesse contexto ${ }^{13}$, fizeram com que se intensificassem os esforços para otimizar os fatores preditivos para o desfecho da extubação. A capacidade dos tradicionais indicadores de desmame (freqüência respiratória, volume corrente, pressão inspiratória máxima e razão entre freqüência e volume corrente) de discriminar entre crianças com extubação bem-sucedida e aquelas que exigem reintubação já se mostrou muito limitada 4,5,8,12. As análises multivariáveis indicam que status pré-morbido, gravidade da doença subjacente e complicações associadas à reintubação não se correlacionam com o aumento da mortalidade por falha de extubação. Uma hipótese alternativa é a deterioração clínica que ocorre entre o momento da extubação e o restabelecimento da ventilação. É nessa direção que se estabeleceu uma relação entre mortalidade e reintubação $\operatorname{tardia}^{12}$.

Por aproximadamente 50 anos, os médicos intensivistas que tratam adultos têm investigado o papel da relação espaço morto/volume corrente (VD/VT) em diversos cenários clínicos. Contudo, essa relação nunca foi usada como 
indicador para desmame da ventilação mecânica, talvez porque fosse uma medida muito trabalhosa - antes do desenvolvimento de métodos mais novos, automatizados (como o $\mathrm{CO}_{2} \mathrm{SMO}$ usado por Bousso et al. ${ }^{14}$ ). Outra razão, como apontaram Hubble et al. ${ }^{11}$, poderia ser o fato de que a doença pulmonar crônica é muito comum em adultos e, nesse caso, o médico precisaria ter informações sobre o status respiratório anterior à doença aguda, o que impossibilitaria a aplicação do VD/VT. Por outro lado, a maioria das crianças têm função pulmonar basal normal, e, conseqüentemente, uma relação VD/VT normal antes do estabelecimento da doença.

Considerando os índices de desmame previamente empregados, e já que não há um indicador único ou fórmula que tenha se mostrado útil para prever a falha de extubação em crianças, Bousso et al. ${ }^{14}$ conduziram um estudo prospectivo usando a relação VD/VT como marcador de maturidade para extubação em crianças com VM. Até agora, o único precedente de uso do VD/VT como fator preditivo de maturidade para extubação foi um estudo clínico prospectivo feito por Hubble et al. ${ }^{7}$ com 45 pacientes clínicos e cirúrgicos, com idade entre 1 semana e 18 anos, intubados e com VM. Se os pacientes satisfizessem os critérios de extubação, conforme avaliação da equipe médica, eram submetidos a uma prova de suporte de pressão e, depois de 20 minutos, eram extubados. A análise de regressão logística mostrou que o ponto de corte de VD/VT $\leq 0,5$ era um fator preditivo independente do desfecho da extubação, com uma sensibilidade e especificidade de 0,75 e 0,92, respectivamente. Os valores preditivos positivo (VPP) e negativo (VPN) foram de 0,96 e 0,60, respectivamente. Os autores constataram que, além de identificar crianças que teriam sucesso na extubação, a relação VD/VT também era útil para identificar as crianças em risco para falha respiratória após a extubação (VPN de 0,8 para VD/VT $\geq 0,65$ ).

No presente estudo, Bousso et al. ${ }^{14}$ seguem um protocolo semelhante, estabelecendo um ponto de corte de $\mathrm{VD} / \mathrm{VT} \geq 0,65$ para detectar a falha de extubação. Utilizando esse ponto de corte menos específico e mais sensível, os autores diminuíram a probabilidade de identificar pacientes que terão sucesso na extubação (menor VPP), mas aumentaram a probabilidade de selecionar corretamente aqueles em alto risco para falha (maior VPN). Diferentemente dos resultados do estudo anterior, eles não detectaram diferença significativa entre os grupos, com uma relação VD/VT de 0,62 $( \pm 0,17)$ e 0,65 $( \pm 0,21)$ para as crianças com sucesso e falha na extubação, respectivamente. O VPN para prever a falha de extubação foi de $41,9 \%$, contra $80 \%$ no estudo de Hubble et al. ${ }^{11}$.

Ao analisar as circunstâncias que poderiam ter causado essa diferença de resultados, Bousso et al. apontam a idade média de 17 meses em seu estudo em comparação com a média de 43 meses no estudo de Hubble. Outra diferença importante entre os estudos é o predomínio de pacientes com cirurgia eletiva, com pulmões aparentemente normais, na amostra estudada por Hubble, enquanto a população de Bousso incluiu crianças com doença respiratória aguda grave. É digno de nota o fato de que, no momento da hospitalização, havia mais de um diagnóstico para muitas das crianças (142 diagnósticos para 86 pacientes), dificultando a caracterização da amostra. $\mathrm{A} \mathrm{PaO}_{2} / \mathrm{FiO}_{2}$ menor no momento da admissão, como fator independente relacionado à falha de extubação, refletindo uma doença mais grave, está de acordo com estudos anteriores ${ }^{6}$.

Bousso et al. afirmam que a diferença nos resultados poderia ser o reflexo da inclusão de populações com características diferentes entre si. Essa é uma situação comum nos estudos feitos na América Latina, onde há uma predominância de doenças respiratórias primárias 1,5,8-10,14 em comparação a Estados Unidos e Europa, onde predominam os pacientes de cirurgia eletiva $a^{4,6,11,15}$.

Atualmente, ainda não há critérios confiáveis para prever quais pacientes exigirão reintubação após tolerar um teste de ventilação espontânea. Novos estudos devem enfocar a busca por fatores que nos permitam determinar o momento apropriado para a retirada da sedação antes do desmame e extubação e para evitar infecção respiratória após a extubação. O pronto manejo da insuficiência respiratória pós-extubação também é muito importante, Como a ventilação não-invasiva pode ser ineficaz nessa situação, a VM invasiva deve ser reiniciada assim que possível ${ }^{13}$.

\section{Referências}

1. Farias JA, Frutos F, Esteban A, Flores JC, Retta A, Baltodano A, et al. What is the daily practice of mechanical ventilation in pediatric intensive care units? A multicenter study. Intensive Care Med. 2004;30:918-25.

2. Rothaar RC, Epstein SK. Extubation failure: magnitude of the problem, impact on outcomes, and prevention. Curr Opin Crit Care. 2003;9:59-66.

3. Baisch SD, Wheeler WB, Kurachek SC, Cornfield DN. Extubation failure in pediatric intensive care incidence and outcomes. Pediatr Crit Care Med. 2005;6:312-8.

4. Khan N, Brown A, Venkataraman ST. Predictors of extubation success and failure in mechanically ventilated infants and children. Crit Care Med. 1996;24:1568-79.

5. Farias JA, Alia I, Esteban A, Golubicki AN, Olazarri FA. Weaning from mechanical ventilation in pediatric intensive care patients. Intensive Care Med. 1998;24:1070-5.

6. Manczur TI, Greenough A, Pryor D, Rafferty GF. Comparison of predictors of extubation from mechanical ventilation in children. Pediatr Crit Care Med. 2000;1:28-32.

7. Hubble CL, Gentile MA, Tripp DS, Craig DM, Meliones JN, Cheifetz IM. Deadspace to tidal volume ratio predicts successful extubation in infants and children. Crit Care Med. 2000;28: 2034-40.

8. Farias JA, Alia I, Retta A, Olazarri F, Fernandez A, Esteban A, et al. An evaluation of extubation failure predictors in mechanically ventilated infants and children. Intensive Care Med. 2002;28: 752-7.

9. Fontela PS, Piva JP, Garcia PC, Bered PL, Zilles K. Risk factors for extubation failure in mechanically ventilated pediatric patients. Pediatr Crit Care Med. 2005;6:166-70.

10. Farias JA, Retta A, Alia I, Olazarri F, Esteban A, Golubicki A, et al. A comparison of two methods to perform a breathing trial before extubation in pediatric intensive care patients. Intensive Care Med. 2001;27:1649-54.

11. Kurachek SC, Newth CJ, Quasney MW, Rice T, Sachdeva RC, Patel NR, et al. Extubation failure in pediatric intensive care: a multiple-center study of risk factors and outcomes. Crit Care Med. 2003;31:2657-64. 
12. Epstein SK, Ciubotaru RL. Independent effects of etiology of failure and time to reintubation on outcome for patients failing extubation. Am J Respir Crit Care Med. 1998;158:489-93.

13. Esteban A, Frutos-Vivar F, Ferguson ND, Arabi Y, Apezteguia C, Gonzalez M, et al. Noninvasive positive-pressure ventilation for respiratory failure after extubation. N Engl J Med. 2004;350: 2452-60.
14. Bousso A, Ejzenberg B, Ventura AM, Fernandes JC, Fernandes IC, Goes PF, et al. Evaluation of the dead space to tidal volume ratio as a predictor of successful extubation. J Pediatr (Rio J). 2006;82:347-53.

15. Chavez A, dela Cruz R, Zaritsky A. Spontaneous breathing trial predicts successful extubation in infants and children. Pediatr Crit Care Med. 2006;7:324-8. 